\title{
Les groupes thématiques de la Société internationale francophone d'éducation médicale (SIFEM) : des ressources d'expertise et de recherche fécondes pour notre communauté
}

Dans le sillage de plusieurs autres manuscrits déjà publiés, la présente livraison du journal propose à nos lecteurs deux nouvelles contributions élaborées sous l'égide d'un des groupes thématiques de la Société internationale francophone d'éducation médicale (SIFEM).

L'article de Nathalie Loye et Sylvie Fontaine [1] complète le premier qu'elles avaient publié dans un précédent numéro [2] et s'inscrit dans le cadre d'une série promue sous l'égide du groupe thématique «Évaluation et mesure». Elles y font une synthèse pragmatique des dispositifs instrumentaux développés au service de l'évaluation des apprentissages.

Pour sa part, le texte de Laurent Couchana [3] inaugure la série du groupe «Numérique pour la pédagogie» et apporte une information concise sur le développement de Twitter en tant qu'outil de formation professionnelle continue, illustrant les potentialités didactiques et pédagogiques spectaculaires des réseaux sociaux, aujourd'hui encore à peine entrevues.

Des publications émanant d'autres groupes thématiques sont en cours de préparation et seront publiées prochainement, à l'issue d'un processus d'évaluation éditoriale en cours, coordonné par le comité de rédaction.

Chacune à leur manière, ces différentes publications illustrent de façon originale la fécondité des groupes thématiques en tant que structures abritant des ressources d'expertise et de recherche. Mis sur pied dans le cadre de la relance de notre société lors du congrès de Marseille en octobre 2017, leurs mandats sont pluriels. Ils répondent ainsi, notamment, à la préoccupation de la SIFEM d'offrir à ses membres des synthèses didactiques, documentées et actualisées concernant les grandes problématiques qui sont débattues dans le cadre de la formation des divers professionnels de la santé. Ils ont aussi vocation à proposer, dans une perspective exploratoire, des pistes de réflexions susceptibles de soutenir des visées innovantes, en résonance avec ce que seront demain les pratiques en matière d'éducation des professionnels de la santé et des patients. De telles démarches requièrent des collaborations interdisciplinaires et interprofessionnelles, et c'est le rôle des groupes thématiques que de les explorer et de les coordonner.
Le groupe «Mesure et évaluation», dirigé par Éric Dionne, de l'université d'Ottawa, s'est ainsi donné pour mission d'explorer, d'approfondir et de favoriser le développement des connaissances et l'amélioration des pratiques en matière d'évaluation des apprentissages dans les programmes de formation en santé. Ceci inclut la promotion de l'accès, en contexte francophone, à une variété de ressources pour accompagner les évaluateurs et les chercheurs qui s'intéressent à ce thème. Le groupe a déjà publié deux articles dans la revue $[1,2]$; un troisième est en préparation. Le groupe a également animé une session au cours de la première édition des Ateliers d'automne de la SIFEM, à Brest, en octobre 2018; un atelier de niveau avancé, portant sur l'adéquation des outils et pratiques d'évaluation en fonction de l'évolution continue de la formation des professionnels de la santé, sera proposé lors de la deuxième édition de cet évènement, à Nice, en octobre 2019.

Le groupe «Éthique des curriculums de formation en santé », piloté par Florence Parent, de l'Université libre de Bruxelles, expérimente résolument l'interdisciplinarité et l'interprofessionnalité, convoquant, entre autres, la philosophie, l'épistémologie ou les sciences de l'éducation pour requestionner la généalogie des sciences cliniques et des pratiques professionnelles en santé, au travers d'un examen critique des dispositifs curriculaires et pédagogiques en place dans le cadre de la formation des divers professionnels de la santé. Il a également proposé une session lors de la première édition des Ateliers d'automne de la SIFEM. Il développe sa réflexion lors de regroupements périodiques de ses membres et se prépare à partager sa réflexion sous la forme de publications ou lors des prochains évènements francophones annuels d'éducation médicale.

Le groupe «Numérique pour la pédagogie » est animé par Nicolas Picard, de l'Université de Limoges. Il s'est donné pour mission principale de mener une réflexion sur les usages du numérique dans les formations en santé. Pour ce faire, le groupe de travail se propose de travailler sur une typologie des usages du numérique en pédagogie des sciences de la santé, dans le but de favoriser la diffusion et l'évaluation des approches ou des outils les plus pertinents. 
Les évolutions dans ce domaine étant constantes, cette typologie sera dynamique. Une dimension interprofessionnelle sera recherchée pour faciliter la transposition de concepts d'un domaine des sciences de la santé à un autre. La typologie intégrera une dimension analytique destinée à objectiver l'intérêt des dispositifs. Le groupe publiera régulièrement dans Pédagogie Médicale des articles décrivant de nouveaux outils utiles aux enseignants des formations professionnelles en santé.

Le groupe «Communication professionnelle en santé», piloté par Marie Thérèse Lussier, de l'Université de Montréal, s'engage à réfléchir sur les différentes formes que peut prendre la communication en santé et les modalités de formation les plus aptes à permettre l'apprentissage par les professionnels d'une communication adéquate avec leurs patients, leurs collègues et le public. Ainsi, les principales catégories qui seront discutées sont la communication interpersonnelle, qui inclut la communication patient-médecin, la communication avec la famille et les proches, ainsi que la communication entre pairs et avec les autres intervenants en santé. Le groupe se propose notamment d'expliciter les aspects relationnels et informationnels associés à la communication professionnelle, en donnant également une place importante aux stratégies de formation et d'évaluation les mieux adaptées pour préparer les futurs professionnels de la santé à une communication professionnelle de qualité.

Le groupe «Raisonnement clinique» est animé par Marie-Claude Audétat, de l'Université de Genève. Il s'est donné pour mandat de faire le lien entre les données documentées (evidences) disponibles dans la littérature sur le raisonnement clinique et leurs implications pratiques dans les contextes d'enseignement et de supervision clinique. Dans cette perspective, il aborde l'enseignement du raisonnement clinique selon des angles différents, liés par exemple aux usages de la simulation qui se développent, aux enjeux éthiques qui émergent, aux questions que soulèvent la collaboration interprofessionnelle, le suivi des maladies chroniques, la régulation de qualité des soins ou la gestion des erreurs. Il s'intéresse également aux conditions opérationnelles à mettre en place pour promouvoir l'enseignement et l'apprentissage précoces du raisonnement clinique dans les curriculums de formation.

Enfin le groupe «Responsabilité sociale des institutions de formation », qui est né au sein de la SIFEM, a pris son essor en devenant un mouvement autonome très dynamique, fédéré sous l'égide du Réseau international francophone pour la responsabilité sociale en santé (RIFRESS), dont le congrès s'est tenu à Rabat au Maroc, en mars dernier.

Les groupes thématiques sont soutenus institutionnellement par la SIFEM, qui s'efforce de mettre à leur disposition des outils d'échanges et de communication, des espaces d'archivage et de gestion de ressources, mais aussi de faciliter leurs réunions et de promouvoir la diffusion de leurs productions. N'hésitez pas à prendre contact avec leurs responsables pour toute forme de projet en lien avec leurs thèmes respectifs (https://www.sifem.net/fr/grou pes-thematiques): sollicitation d'une expertise scientifique ou d'un avis sur un projet pédagogique, participation à une manifestation ou invitation à un partage d'expérience, etc. Interrogez-les sur les conditions permettant de rejoindre respectivement chacun des groupes ou d'être associé à leurs travaux.

Les objectifs des groupes thématiques sont associés à des défis importants. En lien avec tous les partenaires de la SIFEM, il s'agit notamment de participer à l'élaboration des différents corpus francophones de référence en éducation pour les sciences de la santé. Au regard des ancrages culturels irréductibles et des transpositions contextuelles nécessaires, ceux-ci ne peuvent en effet résulter d'une simple traduction des productions anglo-saxonnes. Il s'agit ainsi d'une implication qui engage socialement notre communauté, ce qui n'est pas un mince enjeu.

$$
\begin{array}{r}
\text { Bernard CHARLIN } \\
\text { Président de la SIFEM } \\
\text { Nicolas FERNANDEZ } \\
\text { Rédacteur en chef-adjoint de Pédagogie Médicale } \\
\text { Jean JOUQUAN } \\
\text { Rédacteur en chef de Pédagogie Médicale }
\end{array}
$$

*Mailto : jean. jouquan@univ-brest.fr

\section{Références}

1. Loye N, Fontaine S. S'instrumenter pour évaluer. Pédagogie Médicale 2018;19:95-107.

2. Fontaine S, Loye N. L'évaluation des apprentissages: une démarche rigoureuse. Pédagogie Médicale 2017:18:189-98.

3. Chouchana L, Auffret M, Geniaux H. Twitter ${ }^{\text {, }}$, un outil de formation médicale continue? Pédagogie Médicale 2018;19:91-93. 